\title{
Traumatic Dislocation of Implanted Collamer Phakic Lens: A Case Report and Review of the Literature
}

\author{
Majid Moshirfar ${ }^{*}$, Brian C. Stagg, Valliammai Muthappan and Shail A. Vasavada
}

John A. Moran Eye Center, University of Utah School of Medicine, USA

\begin{abstract}
Purpose: Present a case of dislocation of an Implantable Collamer Lens (ICL) that occurs after blunt trauma and review the published literature on this topic.

Methods: Case report and literature review using PubMed.

Results: A 44 year-old male presented to the emergency department with sudden onset of blurry vision after blunt trauma to the left eye. Three years prior, he had undergone bilateral ICL placement for high myopia. On examination, the superotemporal haptic was noted to be dislocated into the anterior chamber, but there was no endothelial touch by the dislocated lens. The patient had an associated hyphema that was medically managed, and 2 weeks after the injury, the patient underwent ICL repositioning. A review of the literature was performed and five previous cases of ICL dislocation were identified. Three of these occurred after blunt trauma. One of these cases was associated with endothelial touch and decompensation and eventually required a descement's stripping endothelial keratoplasty (DSAEK).

Conclusion: Dislocation of ICLs after blunt trauma is a rare but important potential sequela of the procedure and can lead to corneal decompensation if there is lens-endothelial touch. All patients who suffer blunt dislocation of ICL should be followed long-term after repositioning for signs of endothelial loss and dysfunction.
\end{abstract}

Keywords: Dislocation, high myopia, ICL, implantable collamer lens, IOL, lens dislocations, myopia, phakic IOL, PIOL, staar ICL, trauma, traumatic lens dislocation, vision.

\section{INTRODUCTION}

Phakic intraocular lenses ( $p$ IOLs) have been used for decades in refractive surgery, particularly for patients with very high correction [1]. Multiple styles are available, including iris fixated anterior chamber PIOLs, angle fixated anterior chamber PIOLs, and posterior chamber PIOLs. The Implantable Collamer Lens (ICL; Staar Surgical, Monrovia, California, USA) is a phakic IOL that is placed in the posterior chamber without any fixation. Long term studies have generally found these lenses to be efficacious and safe $[2,3]$. Here we present a case report of ICL dislocation after blunt trauma and a review of the previously reported cases of ICL dislocation in general.

\section{CASE REPORT}

A 44 year-old man presented to the emergency department with blurry vision in the left eye after being struck in the eye with a pipe. The patient had bilateral ICLs placed for high myopia three years prior to presentation and had no previous complications associated with this surgery. The patient reported mild pain associated with brow and lid abrasions from the injury. In the left eye, his visual acuity at presentation was hand motions and intraocular pressure (IOP) was 22. He had 4+ red blood cells in the anterior

\footnotetext{
*Address correspondence to this author at the John A. Moran Eye Center, University of Utah School of Medicine, 65 Mario Capecchi Drive, Salt Lake City, Utah 84132, USA; Tel: (801) 581-2352;

E-mail: majid.moshirfar@hsc.utah.edu
}

chamber (AC) with a blood clot located inferiorly. The superotemoporal haptic of the ICL was dislodged into the $\mathrm{AC}$ and entrapped in the pupillary aperture; the remaining haptics were behind the iris (Fig. 1). There was associated pigment clumping on the ICL. Fundoscopic exam revealed no abnormalities. There was no endothelial touch by the prolapsed ICL. The patient's right eye visual acuity (VA) was $20 / 20$ and had no abnormalities on examination other than a shallow vault between the lens and the ICL (50\% of corneal thickness).

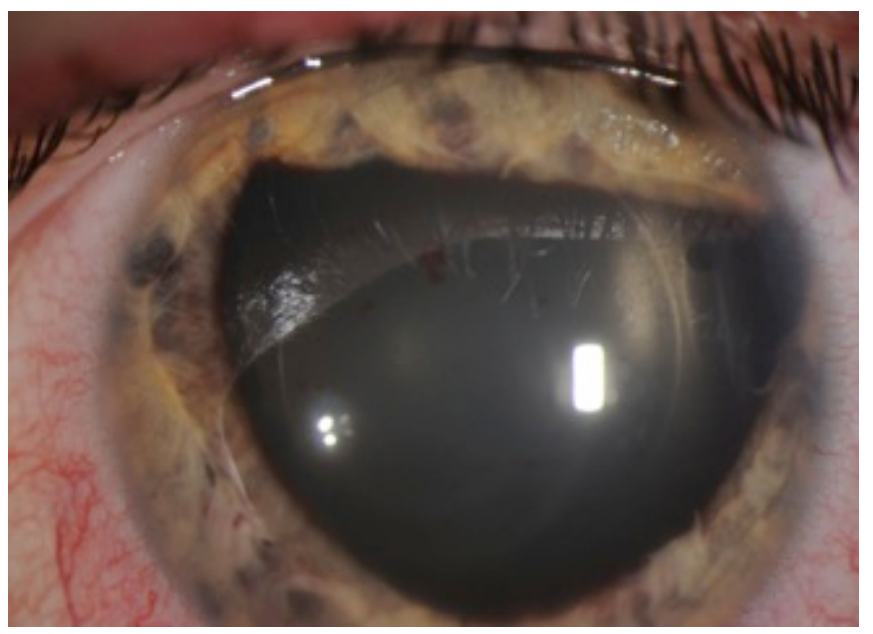

Fig. (1). Slit lamp photograph of traumatic ICL dislocation. Superotemporal footplace dislocated into the anterior chamber with associated peaking of the pupil at the edges of the 1CL. 
The patient was started on prednisolone acetate $1 \%$ eye drops (Allergan, Irvine, CA, USA) and atropine suflate 1\% eye drops (Bausch and Lomb, Rochester, NY, USA) to the left eye. As the hyphema cleared, the vision improved to 20/30 in the left eye. Two weeks after injury, the ICL was repositioned without complication. Earlier repositioning was not possible due to the patient's social circumstances. His post-procedure visual acuity was 20/20.

\section{DISCUSSION}

Phakic IOLs are commonly used as an alternative to laser refractive surgery with the advantages of leaving the ventral cornea untouched, inducing fewer higher order aberrations, and resulting in better optical and visual quality [1, 3, 4]. Additionally, refractive correction up to $-21.0 \mathrm{D}$ is possible, accommodation is preserved, and implantation is potentially reversible. The ICL is an extremely thin intraocular lens with four plate haptics (foot processes) that is implanted in the ciliary sulcus. The ICL has a specially designed central vault which prevents contact between the ICL and the anterior lens capsule.

The complications associated with ICLs are rare and include early or late anterior subacpsular cataract, increase in intraocular pressure (IOP), angle closure glaucoma, transillumination defects, retinal detachments and corneal endothelial cell failure. Initial studies regarding the ICL did not report dislocation as a significant concern $[4,5]$. One case report even describes a case in which ICLs were stable after a grenade explosion [6]. Subsequently, there have been five cases of ICL dislocation reported in the literature [7-11], three of which were caused by blunt ocular trauma, similar to ours (Table 1) [7, 10, 11].

Kaufer et al. reported a case of spontaneous dislocation into the vitreous cavity secondary to zonular dehiscence. The two inferior ICL footplates subluxed through ruptured zonules into the vitreous cavity. With no history of trauma, possible causes for this dislocation included eye rubbing, improper sizing of the ICL, and inherent zonular pathology. Ultimately, the lens was explanted. In a second case, reported by Kong et al. in 2010, dislocation occurred after the patient suffered severe occipital trauma in a dark room. The patient's pupil was likely large due to scoptic conditions, and the posterior trauma forced the infero-nasal footplate anteriorly into the AC.

There have been three previously reported cases of ICL dislocation caused by blunt ocular trauma $[7,10,11]$. The patients were aged 26, 33, and 39. Two of them were males and one was a female. One of the patients was struck by a

Table 1. Review of cases of ICL dislocation after trauma.

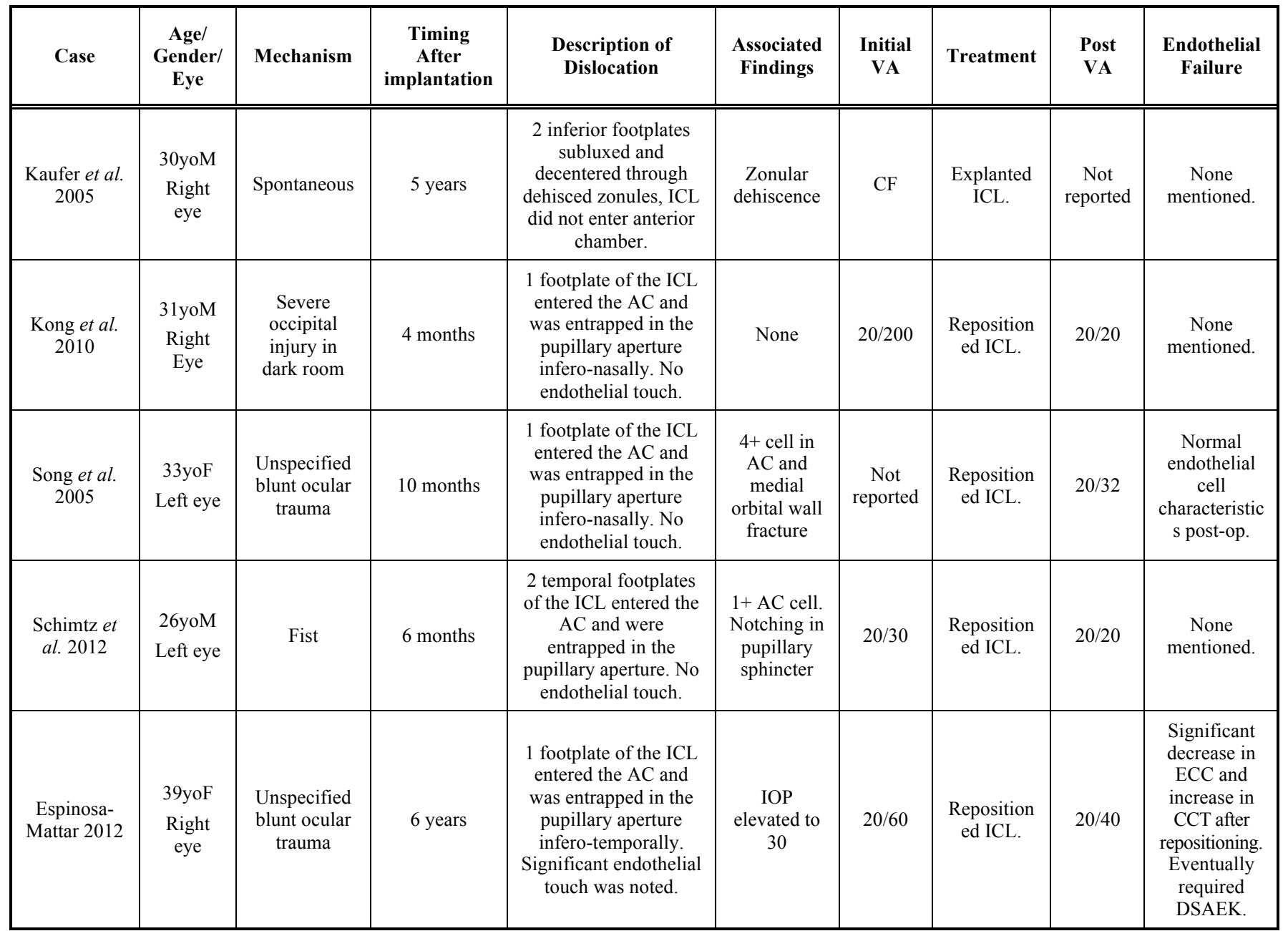

$\mathrm{AC}=$ anterior chamber. $\mathrm{CCT}=$ Central corneal thickness. $\mathrm{CF}=$ count fingers. ECC $=$ Endothelial cell count. ICL $=$ Implantable Collamer Lens $($ Staar Surgical, Monrovia, California, USA). IOP $=$ Intraocular pressure. 
fist, and the mechanism of injury was not specified in the others. Two of the three reported patients were symptomatic with blurry vision [7, 11]. Interestingly, one of the patients was asymptomatic and the dislocated IOL was only discovered on a regularly scheduled eye exam two weeks after his blunt injury; his vision had decreased to 20/30 from 20/20 [10]. One case reported associated hyphema [11]. In one case the infero-nasal footplate was dislocated [1], in another the infero-temporal footplate [7], and in the third the two temporal footplates were dislocated [10].

All three of the reported patients were treated with repositioning of the ICL within one week of presentation $[7,10,11]$. Our patient underwent ICL repositioning two weeks after the inciting event; due to the patient's social circumstances, an earlier surgery was not possible. One of the three reported significant lens-endothelial touch at presentation and 20/60 initial VA [7]. Three months after repositioning, this patient developed bullous keratopathy with central corneal thickness 653 microns and endothelial cell count of less than 800 cells $/ \mathrm{mm}^{2}$. Ultimately, DSAEK was required in this case.

\section{CONCLUSION}

A review of the cases of ICL dislocation reveals that it is a rare but real long term complication of the procedure, particularly after trauma. There is no pattern as to which footplate is dislocated. While reports suggest patients do well with repositioning of the lens, one of the most concerning sequlae is endothelial decompensation, which occurred in one of five previously reported eyes. This case presented with lens-endothelial touch, which was likely the causative factor of the corneal decompensation, rather than endothelial trauma from repeat surgery. Interestingly, some of the patients with dislocation did not have significantly reduced visual acuity. However, while the patient may be satisfied with this level of vision, early repositioning of the ICL is necessary to avoid further complications.

\section{CONFLICT OF INTEREST}

No author has a financial or proprietary interest in any material or method mentioned.

\section{ACKNOWLEDGEMENTS}

\author{
Declared none.
}

\section{REFERENCES}

[1] Olson RJ, Werner L, Mamalis N, et al. New intraocular lens technology. Am J Ophthalmol 2005; 140(4): 709-16.

[2] Koivula A, Petrelius A, Zetterstrom C. Clinical outcomes of phakic refractive lens in myopic and hyperopic eyes: 1-year results. J Cataract Refract Surg 2005; 31(6): 1145-52.

[3] Sanders DR, Vukich JA, Doney K, et al. U.S. Food and Drug Administration clinical trial of the Implantable Contact Lens for moderate to high myopia. Ophthalmology 2003; 110(2): 255-66.

[4] Brown DC, Grabow HB, Martin RG, et al. Staar Collamer intraocular lens: clinical results from the phase I FDA core study. J Cataract Refract Surg 1998; 24(8): 1032-8.

[5] Brown DC, Ziemba SL. Collamer intraocular lens: clinical results from the US FDA core study. J Cataract Refract Surg 2001; 27(6): 833-40.

[6] McCauley MB, Anderson DM, Johnson AJ. Posterior chamber visian implantable collamer lens: stability and evaluation following traumatic grenade explosion. J Refract Surg 2008; 24(6): 648-51.

[7] Espinosa-Mattar Z, Gomez-Bastar A, Graue-Hernandez EO, et al. DSAEK for implantable collamer lens dislocation and corneal decompensation 6 years after implantation. Ophthalmic Surg Lasers Imaging 2012; 43: e68-72.

[8] Kaufer RA, Kaufer GJ. Late subluxation of an ICL. J Cataract Refract Surg 2005; 31(6): 1254-5.

[9] Kong J, Qin XJ, Li XY, et al. Implantable collamer lens dislocation. Ophthalmology 2010; 117(2): 399 e1.

[10] Schmitz JW, McEwan GC, Hofmeister EM. Delayed presentation of traumatic dislocation of a visian implantable collamer lens. J Refract Surg 2012; 28(5): 365-7.

[11] Song JS, Moon HS, Shyn KH. Pupillary capture of implantable contact lens after blunt trauma. J Cataract Refract Surg 2005; 31(9): 1831-3. 